\title{
Zur Kenntniss des Chemismus der peptischen und tryptischen Verdauung der Eiweissstoffe. \\ Von \\ D. Lawrow.
}

(Aus dem physiol.-chem. Laboratorium der St. Petersburger militär-ärztl. Akademie.)

(Der Redaction zugegangen am 8. December 1898.)

Im Laufe meiner Untersuchungen ${ }^{1}$ ) über den Abbau der Eiweisskörper durch das Pepsin und Trypsin bin ich bezüglich der Verdauungsprodukte, welche durch die Einwirkung der genannten Fermente auf die Eiweisskörper entstehen, zu einer Reihe von Resultaten gelangt, welche ein allgemeineres Interesse beanspruchen.

A. Verdaungsversuche mit Pepsin.

Versuch I. $2 \mathrm{~kg}$. mit Wasser gewaschenes, abgepresstes Fibrin wurden mit 6 Liter $0,5 \%$ iger Salzsäure + 2 Liter künstlichen dialysirten Magensaftes 4 Tage bei $40-45^{\circ} \mathrm{C}$. gehalten. Der Magensaft, absichtlich schwach wirkend genommen, enthielt $0,61 \%$ Trockensubstanz. Die erhaltene, durch Fliesspapier filtrirte Lösung gab ungefähr $600 \mathrm{gr}$. bei Zimmertemperatur getrockneten Neutralisationsniederschlag und ungefähr 150 gr. durch Ammoniumsulfat nach der Methode von W. Kühne und R. Chittenden ausscheidbarer, bei 100-1050 getrockneter Verdauungsprodukte. Die Untersuchung der mit Thierkohle entfärbten, durch Ammoniumsulfat unfällbaren Ver-

1) D. Lawrow, Zur Frage nach dem Chemismus der peptischen und tryptischen Verdauung der Eiweissstoffe. Inaug.-Diss. Petersburg 1897 (russisch).

Hoppe-Seyler's Zeitschrift f. physiol. Chemie. XXVI. 
dauungsprodukte zeigte, dass dieselben die Adamkiewicz'sche, Pettenkofer'sche ${ }^{1}$ ) und Liebermann'sche ${ }^{2}$ ) Reaction nicht, die Xanthoproteinreaction sehr schwach gaben.

Versuch II. Gut gewaschenes und stark abgepresstes Fibrin (etwa $2 \mathrm{~kg}$.) wurde unter 4 Liter 96\% igem Alkohol eine Woche gehalten, bei $70-80^{\circ} \mathrm{C}$. getrocknet, fein pulverisirt, dann mit 95\% igem kochenden Alkohol, endlich mit kochendem Aether so lange extrahirt, bis der letztere nichts mehr auszog. Das auf angegebene Weise behandelte Fibrin wurde mit 5 Liter $0,5 \%$ iger Salzsäure und 4,5 Liter künstlichen dialysirten Magensaftes unter Zusatz von $15 \mathrm{gr}$. Thymol bei $40-45^{\circ} \mathrm{C}$. so lange stehen gelassen (ca. 3 Wochen), bis eine neutralisirte Probe mit Ferrocyankalium und Essigsäure keine Fällung mehr gab. Der benutzte Magensaft enthielt 0,15\% organische Substanz und $0,5 \%$ Salzsäure. Die durch Fliesspapier filtrirte Lösung wurde so lange mit Aether ausgeschüttelt, als derselbe etwas aufnahim. Den ätherischen Auszügen wurde Wasser zugesetzt, aus dem Gemenge wurde der Aether entfernt, die erhaltene wässerige Lösung mit Thierkohle entfärbt und concentrirt. Die Lösung der Verdauungsprodukte wurde nach dem Extrahiren mit Aether etwas eingedickt, gegen Congo (wässerige Lösung) genau neutralisirt, von dem sehr unbedeutenden Neutralisations-. niederschlage abgetrennt und mit Ammoniumsulfat nach der Methode von $\mathrm{Kühne}$ und Chittenden behandelt. Sowohl die Lösung der durch Ammoniumsulfat niedergeschlagenen, vom genannten Reagens befreiten Substanzen, als auch die Lösung der durch Ammoniumsulfat unfällbaren Verdauungsprodukte wurden eingedickt, durch Alkohol und Aether bei $0^{\circ} \mathrm{C}$. in 6 Fraktionen gefällt. Darauf wurde das specifische Drehungsvermögen der verschiedenen Fractionen ermittelt.

A. Die durch Ammoniumsulfat niedergeschlagenen Verdauungsprodukte lenkten das polarisirte Licht $a b$ :

\begin{tabular}{|c|c|c|c|c|c|}
\hline 1. & 2. & 3. & 4. & 5. & 6. \\
\hline$-49^{\circ}$ & $-51,5^{0}$ & $-49^{\circ}$ & $-50^{\circ}$ & $-54^{0}$ & $-37^{0}$ \\
\hline
\end{tabular}

1) D. h. Rothfärbung mit Schwefelsäure und Rohrzucker.

2) D. h. blaurothe Färbung bei schwachem Erwärmen mit concentrirter Salzsäure. 
B. Die durch Ammoniumsulfat unfällbaren Verdauungsprodukte lenkten das polarisirte Licht ab:

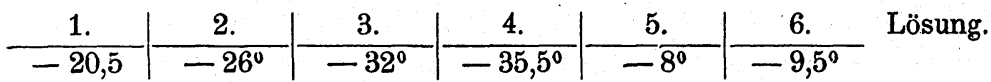

Bei der Untersuchung dieser zwölf Lösungen und der Lösung der durch Aether extrahirten Substanzen fand ich unter Anderem Folgendes.

Alle diese Lösungen gaben die Reactionen von Liebermann, Adamkiewicz und Pettenkofer nicht mehr; die Xanthoproteinreaction war sehr schwach.

Bei der 5. und 6. Lösung der zweiten Gruppe der Verdauungsprodukte blieb ausserdem auch die Millon'sche Reaction aus.

Die Lösungen der ersten Gruppe der Tabelle gaben keine Fällungsreaction mit Ferrocyankalium und Essigsäure.

Die Lösung der durch Aether extrahirten Substanzen zeigte gar keine Biuretreaction und keine Fällungsreaction mit Ammoniumsulfat, dagegen Millon'sche- ${ }^{1}$ ) Xanthoproteinreaction und Fällungsreactionen mit den Alkaloidreagentien.

V ersuch III. $12 \mathrm{~kg}$. der frischen, sorgfältig gewaschenen und vom Schleim und Fettgewebe befreiten Schweinemägen wurden mit 35 Litern $0,6 \%$ iger Salzsäure unter Zusatz von $175 \mathrm{ccm}$. Chloroform und Thymol ungefähr zwei Monate bei $40-45^{\circ} \mathrm{C}$. gehalten. Die zu verdauende Lösung zeigte ununterbrochen deutliche Reaction auf freie Salzsäure und bewahrte den eigenartigen Geruch nach Chloroform. Die Verdauung vollzog sich so energisch, dass eine nach 4 Wochen genommene Probe der neutralisirten Lösung nur einen ganz kleinen Niederschlag beim Sättigen mit Ammoniumsulfat gab. Die Lösung bewahrte bis zum Ende die Fähigkeiten zu verdauen im höchsten Grade; denn eine nach zwei Monaten genommene Probe löste ein etwa gleiches Volumen des in $0,5 \%$ iger Salzsäure gequollenen

1) Diese Reaction kann nicht etwa auf Thymol bezogen werden, da dasselbe beim Eindampfen der Lösung schon vollständig verflüchtigt war. Die Reaction des Thymols mit Millon's Reagens verläuft übrigens auch anders, wie die der Eiweisskörper und ihrer Derivate. 
Fibrins während 8-10 Minuten. Die durch Fliesspapier filtrirte, vollkommen klare, bis zum Verschwinden der Reaction auf freie Salzsäure neutralisirte Lösung wurde auf dem Wasserbade eingedickt. Beim Abkühlen erstarrte sie zum krystallinischen Brei. Der abgetrennte, mit Fliesspapier sehr sorgfältig abgepresste, bei Zimmertemperatur getrocknete krystallinische Niederschlag wog ungefähr $1,5 \mathrm{~kg}$. und war nicht mehr hygroskopisch. Das Filtrat von diesem Niederschlage gab beim weiteren Eindampfen noch einen starken krystallinischen Niederschlag. Der mit Alkohol durchgewaschene krystallinische Niederschlag bestand fast vollkommen aus Kugeln und Knollen von Leucin; er zeigte keine Millon'sche Reaction. Das Filtrat von den Krystallen lieferte beim Behandeln mit Ammoniumsulfat einen ganz kleinen Niederschlag. Das Filtrat von letzterem Niederschlage wurde vom Ammoniumsulfat befreit und durch Alkohol und Aether in 7 Fractionen getheilt. Die Untersuchung der mit Thierkohle entfärbten Lösungen ergab folgendes Resultat.

Nur die zwei ersten Lösungen zeigten die Biuretreaction.

Den Substanzen, welche die Biuretreaction gaben, fehlten alle anderen Farbenreactionen der echten Eiweisskörper.

Die Substanzen, bei denen die Biuretreaction ausblieb, zeigten die Millon'sche Reaction.

Alle diese Lösungen gaben keine Fällungsreactionen mit Ammoniumsulfat und mit Ferrocyankalium nach Ansäuern mit Essigsäure, dagegen gaben sie die Reactionen mit den Alkaloidreagentien.

Versuch IV. In einem weiteren Versuch mit peptischen Verdauungsprodukten benutzte ich 400 gr. Witte's Pepton. Dieselben wurden im Wasser zum dicken Syrup gelöst. Die Lösung, mit Salzsäure angesäuert (ohne dass jedoch die Reaction auf freie Salzsäure eintrat), wurde mit 3 Volumen $95 \%$ igen Alkohols gemischt, bei $-12^{\circ}$ bis $-15^{\circ} \mathrm{C}$. ungefähr 24 Stunden stehen gelassen und darauf bei der angegebenen Temperatur filtrirt. Das Filtrat wurde mit Wasser verdünnt, zur Austreibung von Alkohol gekocht, wieder eingedickt und mit Ammoniumsulfat bei $80-85^{\circ} \mathrm{C}$. gesättigt. Die ausgesalzene 
Substanz wurde durch Lösen im Wasser und durch Fällen mit Ammoniumsulfat zweimal gereinigt. Ihre wässerige, stark concentrirte, vom genannten Salze befreite Lösung wurde durch Alkohol und Aether bei $0^{\circ} \mathrm{C}$. in 4 Fractionen getheilt. Die wässerigen Lösungen der drei erhaltenen Niederschläge und das Filtrat vom dritten Niederschlage wurden gekocht, mit Thierkohle entfärbt und concentrirt. Das specifische Drehungsvermögen der drei ersten Lösungen wurde $\mathrm{zu}-63^{\circ}$ bis $-67^{\circ}$ bestimmt, das der vierten $\mathrm{zu}-51^{\circ}$.

Die Untersuchung dieser Lösungen zeigte unter Anderem Folgendes: Die drei ersten Lösungen zeigten alle Farbenreactionen der echten Eiweisskörper, sie wurden mit Natriumchlorid und Essigsäure, Ammoniumsulfat und allen Alkaloidreagentien gefällt, aber nicht mit Ferrocyankalium und Essigsäure; die Substanzen dieser Lösungen enthielten keinen unoxydirten Schwefel.

Die vierte Lösung gab dieselben Fällungsreactionen, wie die ersten, doch fehlten ihr die Liebermann'sche, Adamkiewicz'sche und Pettenk ofer'sche Reactionen.

Schlussfolgerungen aus diesen Versuchen.

In Versuch I-III liess ich Pepsin von ungleicher Stärke, während verschiedener Zeitdauer auf Eiweissstoffe einwirken.

Es ergibt sich aus Versuch I, dass auch bei schwacher peptischer Verdauung schon Substanzen entstehen, die durch Ammoniumsulfat nicht mehr aussalzbar sind; diesen Produkten fehlt immer eine Reihe von Farbenreactionen, die dem echten Eiweiss zukommen.

Versuch II lehrt, dass bei einer langdauernden, ziemlich intensiven Verdauung Substanzen entstehen, welche sich durch Aether ausschütteln lassen. Diesen Körpern fehlt die Biuretreaction vollkommen. Bezüglich der Reactionen dieser Körper verweise ich auf Seite 515.

Ferner lassen sich, wie meine Versuche ergeben, aus den bei intensiver Verdauung entstehenden Verdauungsgemischen Körper isoliren, die durch Ammoniumsulfat fällbar sind, denen aber eine Reihe von Farbenreactionen der echten Eiweisskörper verloren gegangen ist und die sich unter einander durch 
ihr specifisches Drehungsvermögen unterscheiden lassen. ${ }^{1}$ ) Endlich lassen sich solche Körper nachweisen, die durch Ammoniumsulfat unfällbar sind, eine Reihe Farbenreactionen der echten Eiweisskörper nicht mehr besitzen und unter einander verschiedene specifische Drehung zeigen. ${ }^{2}$ ) Das Gemenge dieser Körper bildet wohl hauptsächlich das "Amphopepton» Kühne's. Versuch III endlich zwingt zu der Schlussfolgerung, dass bei einer sehr langdauernden und sehr intensiven peptischen Verdauung Körper entstehen, die alle Farbenreactionen bis auf die Biuretreaction verloren haben. Ausserdem bilden sich dabei Körper, die keine Biuretreaction, dagegen die Fällungsreactionen mit den Alkaloidreagentien zeigen, und weiterhin krystallinische Spaltungsprodukte in nicht unbeträchtlicher Menge. Die letzten Substanzen sind allerdings wohl zum Theil bereits in den Geweben der Magen präformirt vorhanden gewesen, in der Hauptmenge jedoch erst während der Verdauung entstanden.

Versuch IV bezweckte hauptsächlich die Isolirung des Peptons von A. Danilewsky, das die drei ersten durch Alkohol und Aether erzielbaren Fractionen bildete.

B. Verdaungsversuche mit Trypsin.

Versuch V. $2,5 \mathrm{~kg}$. gewaschenes Fibrin blieben mit 2 Litern 0,5\% künstlichen, dialysirten pancreatischen Saftes, welcher $0,5 \%$ kohlensaures Natron enthielt, unter Zusatz von $15 \mathrm{gr}$. Thymol ungefähr 2 Wochen bei $40-45^{\circ} \mathrm{C}$. stehen. Der pancreatische Saft enthielt $0,23 \%$ getrocknete organische Substanz und $0,1 \%$ Asche. Die erhaltene Lösung wurde filtrirt und mit Phosphorwolframsäure in Gegenwart von Schwefelsäure (bis zu $5 \%$ ) niedergeschlagen. Der Niederschlag wurde mit $5^{\circ} \%$ iger Schwefelsäure ausgewaschen und mit Barytoxydhydrat zerlegt. Die von Barythydrat durch Schwefelsäure befreite und bis zur syrupösen Consistenz eingedickte Lösung wurde durch Fällung

1) In meinem Versuche II handelte es sich wahrscheinlich um ein Gemenge von zwei Körpern.

2) In meinem Versuch II handelte es sich wahrscheinlich um ein Gemenge von vier Körpern. 
mit Alkohol und Aether bei etwa $0^{\circ} \mathrm{C}$. in vier Fractionen getheilt. Die Untersuchung dieser durch Thierkohle entfärbten Portionen erwies Folgendes.

Die drei ersten Lösungen zeigten Biuretreaction, die vierte dagegen gab dieselbe nicht mehr. Alle diese Lösungen gaben die Adamkiewicz'sche, Pettenkofer'sche, Liebermann'sche und Millon'sche Reaction gar nicht, die Xanthoproteinreaction war sehr schwach. Alle Lösungen gaben bei der Sättigung entweder mit Ammoniumsulfat oder mit Chlornatrium und mit Ferrocyankalium und Essigsäure keine Niederschläge, dagegen mit den Alkaloidreagentien starke Fällungsreactionen.

Versuch VI. $2 \mathrm{~kg}$. des frischen Pancreas wurden mit 3 Litern $0,5 \%$ igen kohlensauren Natrons unter Zusatz von Thymol bei $35-40^{\circ} \mathrm{C}$. ungefähr 3 Tage digerirt. Die filtrirte Lösung, welche starke Biuretreaction zeigte, wurde unter Zusatz von Thymol etwa zwei Monate bei $40-45^{\circ} \mathrm{C}$. digerirt, dann eingedickt, vom krystallinischen Niederschlage befreit und mit Thierkohle entfärbt. Die erhaltene, stark concentrirte Lösung zeigte keine Biuretreaction.

Schlussfolgerungen. Versuch V lehrt, dass eine Lösung der Produkte einer tryptischen Verdauung von Eiweissstoffen, welche durch Phosphorwolframsäure und Schwefelsäure fällbar, jedoch durch Ammoniumsulfat nicht fällbar ist, ein Gemenge darstellt. Dasselbe enthält sogar Substanzen, die keine Biuretreaction mehr zeigen.

Versuch VI endlich zeigt die Möglichkeit durch energische Trypsinwirkung die Proteinkörper völlig zu zersetzen.

Hedin hat bekanntlich aus den pancreatischen Verdauungsprodukten Lysin isolirt. ${ }^{1}$ )

Fr. Kutscher hat zuerst darauf aufmerksam gemacht, dass der Phosphorwolframsäureniederschlag des Antipeptons hauptsächlich aus den Hexonbasen ${ }^{2}$ ) besteht. ${ }^{3}$ )

1) Du Bois-Reymond's Archiv, Jahrgang 1891, S. 248-278.

2) A. Kossel, Diese Zeitschrift, Bd. XXV, S. 165-189.

3) Diese Zeitschrift, Bd. XXV, S. 195-201; id. Bd. XXVI, S. 110-122. 
L. Morochowetz erreichte mit einem dem meinen ähnlichen Verfahren dieselben Erfolge wie ich mit Versuch VI. ${ }^{1}$ )

\section{Wirkung des Labferments.}

Versuch VII. A. Danilewsky hat gefunden, dass das Labferment die wässerigen Lösungen einiger Produkte der peptischen oder tryptischen Verdauung der Eiweisskörper coagulirt, indem es in denselben einen Niederschlag hervorruft, welcher die Eigenschaften der coagulirten Proteinsubstanzen besitzt. Diese Wirkung des Labferments wurde von B. Okuneff auf Veranlassung von A. Danilewsky zuerst ausführlicher untersucht. ${ }^{2}$ ) Ich habe die Untersuchung dieser Wirkung des Labferments auf die Produkte der peptischen und tryptischen Verdauung, welche sich nicht mit Ammoniumsulfat nach der Methode von Kühne und Chittenden fällen lassen, ausgedehnt. Ich benutzte entweder den Reval'schen Labfermentauszug, oder eine wässerige Lösung von Witte's oder Grübler's Labferment. Dabei ergab sich, dass die wässerigen 4-8\% igen, entweder amphoter, oder sauer, oder schwach alkalisch reagirenden Lösungen der letztgenannten Substanzen, mit Labferment gemischt, bei $40-45^{\circ} \mathrm{C}$. unverändert blieben.

Unter den gleichen Bedingungen reagirten die wässerigen Lösungen des Peptons von A. Danilewsky und dieselben von Witte's Pepton stark.

D. Wirkung des trockenen Erhitzens.

Versuch VIII. Es ist seit lange bekannt, dass die verschiedenen Verdauungsprodukte beim Trocknen bis zum constanten Gewicht eine Art Dehydratation erfahren.

Für die folgenden Versuche benutzte ich zunächst wässerige Lösungen des Peptons von A. Danilewsky, die sauer reagirten, aber keine freie Salzsäure enthielten.

Versuch VIIIa. Die wässerige Lösung des Peptons von

1) L. Morochowetz, Die Gesetze der Verdauung, 1881, russisch.

2) Inaug.-Dissert., russisch, 1896; Maly's Jahresb. d. Thierchemie Bd. XXV, S. 291. («Die Bedeutung des Labferments [des Chymosins] bei den Assimilationsprocessen des Organismus», vorläufige Mittheilung.) 
A. Danilewsky wurde auf dem Wasserbade eingedampft und im doppelwandigen, mit Wasser gefüllten Trockenschrank 8 Wochen bei $100^{\circ}$ C. bis zum constanten Gewicht getrocknet. Die auf diese Weise getrocknete Substanz löste sich in kaltem Wasser vollkommen, aber diese Lösung gab die Fällungsreactionen mit Salpetersäure, Kupfersulfat, Ferrocyankalium und Essigsäure, während das Ausgangsmaterial diese Reactionen natürlich nicht zeigte.

Versuch VIIl ${ }^{b}$. Die wässerige Lösung des Peptons von A. Danilewsky wurde auf dem Wasserbade eingedampft; die 4 Tage bei $100^{\circ} \mathrm{C}$. getrocknete Substanz ungefähr 60 Tage bei 105 bis $110^{\circ}$ stehen gelassen. Nach dieser Behandlung löste sie sich in kaltem und heissem Wasser nur in Spuren.

Versuch VIIIc. Die wässerige Lösung des Peptons von A. Danilewsky wurde auf dem Wasserbade eingedampft. Die 3 Tage lang bei $100^{\circ} \mathrm{C}$. getrocknete Substanz wurde 3 Tage bei $120-125^{\circ}$ C. gehalten. Die auf diese Weise getrocknete Substanz löste sich im kalten und heissen Wasser nur theilweise.

Versuch VIIId. Die wässerige Lösung desselben Peptons wurde auf dem Wasserbade eingedampft; die Substanz, 5 Tage lang bei $100^{\circ} \mathrm{C}$. gehalten, wurde ungefähr 7 Wochen bei 105 bis $110^{\circ} \mathrm{C}$. bis zum fast constanten Gewicht getrocknet. Das Gewicht der 5 Tage bei $100^{\circ}$ C. getrockneten Substanz betrug 0,7036 gr.; das Gewicht der bei 105 bis $110^{\circ}$ C. getrockneten Substanz betrug 0,690 gr. Die auf angegebene Weise behandelte Substanz löste sich im Wasser nur theilweise. Der in Wasser unlösliche Rest wurde mit kaltem und heissem Wasser, Alkohol und Aether gewaschen, darauf bei $100^{\circ} \mathrm{C}$. bis zum constanten Gewicht getrocknet. Ich erhielt so 0,3776 gr. = $\frac{0,3776}{0,7036} \times 100=53,66 \%$ des Gewichtes der bei $100^{\circ} \mathrm{C}$. $g \theta$ trockneten Substanz. Die in Wasser unlöslichen Substanzen, welche bei diesen Versuchen erhalten wurden, lösten sich auch beim Erwärmen nur sehr wenig in verdünnten und concentrirten Mineralsäuren, leichter in Aetzalkali. Die alkalischen Lösungen zeigten die Biuretreaction; die Substanzen selbst 
gaben die Millon'sche, Pettenkofer'sche und Adamkiewicz'sche Reaction.

Diese Versuche zeigten, dass das Pepton von A.D anilew sky sehr leicht beim Trocknen bis zum constanten Gewicht, besonders über $100^{\circ}$ G., dehydratirt wird.

Weitere Versuche wurden mit den durch Ammoniumsulfat nicht fällbaren Substanzen angestellt. Trocknete ich dieselben bei $105-110^{\circ} \mathrm{C}$. bis fast zum constanten Gewicht, so wurden sie verändert. Einige von ihnen hatten ihre Löslichkeit in Wasser fast ganz bewahrt, andere, nämlich die Substanz der zwei letzten Fractionen des Versuches II (s. S. 515), hingegen nicht. Diese hatte wahrscheinlich eine tiefgreifende Zersetzung beim Trocknen erfahren, denn aus ihr hatten sich zuerst saure, dann alkalische Dämpfe entwickelt und die Gewichtsabnahme war sehr bedeutend. Das Gewicht der 4 Tage lang bei $100^{\circ} \mathrm{C}$. getrockneten Substanz sank nämlich von 2,0265 gr. während des achtwöchentlichen Trocknens bei $105-110^{\circ} \mathrm{C}$. auf $0,8825 \mathrm{gr}$. Der in Wasser unlösliche Rest betrug in diesem Falle nur noch $11,05^{\circ} \%$ des Gewichtes der 4 Tage lang bei $100^{\circ} \mathrm{C}$. getrockneten Substanz.

Die wässerigen Lösungen der getrockneten Substanzen gaben die Fällungsreactionen mit Kupfersulfat, Ferrocyankalium und Essigsäure und mit Ammoniumsulfat, welche dem Ausgangsmaterial fehlten.

Da nach vorstehenden Versuchen also die mit Ammoniumsulfat unfällbaren Verdauungsprodukte der echten Eiweisskörper weder durch Labferment, noch durch Trocknen in die echten Eiweisskörper sich verwandeln, und da sie zudem nicht alle Farbenreactionen der echten Eiweisskörper zeigen, so muss man sie als Zersetzungsprodukte der Eiweisskörper betrachten. Im Allgemeinen ist eine Lösung der letztgenannten Verdauungsprodukte eine Mischung von Substanzen, deren Zahl und chemische Eigenschaften von Dauer und Intensität der Verdauung abhängig sind.

Alle diese Versuche zeigen, dass das Pepton von A. Danilewsky das äusserste Glied in der Kette der durch die proteolytischen Fermente des Körpers zu erhaltenden Abbau- 
produkte der Eiweisskörper ist, dem alle Farbenreactionen der Eiweisskörper zukommen und das sich durch Labferment oder Trocknen wieder in einen e chten Eiweisskörper überführen lässt.

Dem von A. Danilewsky angegebenen Schema zufolge ist zwischen einem genuinen Eiweisskörper und seinem Peptone eine Reihe von $Z_{\text {wischenprodukten }}{ }^{1}$ ) vorhanden.

Zum Schluss spreche ich meinem verehrten Lehrer Herrn Professor A. Danilewsky, auf dessen Veranlassung und unter dessenLeitung diese Arbeit ausgeführt ist, meinen besten Dank aus.

1) A. Danilewsky, Journ. der russ. physiko-chem. Gesell., Bd. XI und XII (Jahrgang 1880). 